as common $\mathrm{V} \gamma 9 \mathrm{~V} \delta 2-\mathrm{T}$ cell receptor sequence variations. In combination with the conserved monomorphic nature of the V $\gamma 9 \mathrm{~V} 82-\mathrm{TCR}$ and the facile replacement of the tumor-specific $\mathrm{VHH}$, this immunotherapeutic approach can in principle be applied to a large group of cancer types.

Disclosure Information L.A. King: None. R. Lameris: None. R.C. Roovers: None. P. Parren: None. T.D. de Gruijl: None. H.J. van der Vliet: None.

\section{GEMCITABINE INDUCES PRO-APOPTOTIC BH3 ONLY PROTEINS AND SENSITIZES PANCREATIC DUCTAL ADENOCARCINOMA CELLS FOR RLH-TRIGGERED IMMUNOGENIC CELL DEATH}

\begin{abstract}
${ }^{1} \mathrm{P}$ Metzger*, 1,2 HT Bourhis, ${ }^{1,3} \mathrm{M}$ Stieg, ${ }^{1,2} \mathrm{D}$ Böhmer, ${ }^{1} \mathrm{~S}$ Endres, 1,4 P Düwell, 'LM König, ${ }^{1} \mathrm{M}$ Schnurr. ${ }^{1}$ Center of Integrated Protein Science Munich (CIPSM) and Division of Clinical Pharmacology, University Hospital, LMU Munich, Munich, Germany; ${ }^{2}$ Department of Medicine II, University Hospital, LMU Munich, Munich, Germany; ${ }^{3}$ Department of Medicine IV, University Hospital, LMU Munich, Munich, Germany; ${ }^{4}$ Institute of Innate Immunity, University of Bonn, Bonn, Germany
\end{abstract}

10.1136/jitc-2020-ITOC7.13

Background Despite tremendous effort, the prognosis of patients with pancreatic ductal adenocarcinoma (PDAC) remains poor and therapy options are limited. Recent advances in chemotherapeutic schemes have increased the survival of PDAC patients by a few months only. So far, the success of immunotherapy seen in other cancer types could not be transferred to PDAC. Our group has demonstrated that single agent RIG-I-like helicase (RLH)-targeting immunotherapy induces an anti-tumoral immune response and improves survival in a PDAC mouse model dependent on the induction of immunogenic cell death. In addition, we and others were able to show that tumor cell death induction by RLH ligands is partially dependent on the induction of the pro-apoptotic $\mathrm{BH} 3$-only proteins PUMA and NOXA. In the current study we aim at improving therapy response using a combinatorial chemo-immunotherapy (CIT) approach.

Methods Tumor cell death induction by gemcitabine, oxaliplatin and 5-fluoruracil (5-FU) alone or in combination with $\mathrm{RLH}$ ligands was evaluated in the murine cell line Panc02. The induction of PUMA and NOXA was measured by realtime PCR. The capability of chemo-immunotherapy -induced tumor cell death to activate splenic $\mathrm{CD} 8 \mathrm{a}^{+}$dendritic cells (DC) as well as to induce antigen uptake and cross-presentation was investigated in vitro. Therapeutic efficacy was evaluated in vivo using an orthotopic PDAC mouse model.

Results Gemcitabine, oxaliplatin and 5-FU induced dosedependent tumor cell death in vitro. however, only gemcitabine lead to an induction of the pro-apoptotic proteins PUMA and NOXA. Simultaneous treatment with gemcitabine and RLH-ligand increased cell death induction without affecting the cytokine secretion substantially. $\mathrm{CD}^{+} \mathrm{a}^{+} \mathrm{DC}$ activation upon RLH-therapy was not affected by chemotherapy. Of note, antigen uptake as well as $\mathrm{T}$ cell priming was increased by chemo-immunotherapy. Most importantly, the survival of orthotopic PDAC bearing mice was significantly prolonged in the chemo-immunotherapy group compared to single agent treatment.

Conclusions Gemcitabine treatment of PDAC induces PUMA and NOXA expression which leads to mitochondrial priming and sensitization towards RLH-induced cell death. chemo- immunotherapy increases the cross-presentation capability of DC in vitro and prolongs the survival of PDAC bearing mice. chemo-immunotherapy is therefore an attractive combinatorial therapeutic approach in PDAC.

Funding The project was supported by the Deutsche Forschungsgemeinschaft (DFG) - Projektnummer 179062510 and 329628492 - SFB 1321 as well as the Förderprogramm für Forschung und Lehre (FöFoLe) funded by the Ludwig-Maximilians-Universität München.

Disclosure Information P. Metzger: None. H.T. Bourhis: None. M. Stieg: None. D. Böhmer: None. S. Endres: None. P. Düwell: None. L.M. König: None. M. Schnurr: None.

\section{E-Poster Presentations}

\section{P01 Emerging concepts/novel agents}

\section{P01.01 A PHASE 1A/1B DOSE-ESCALATION STUDY OF INTRAVENOUSLY ADMINISTERED SB 11285 ALONE AND IN COMBINATION WITH NIVOLUMAB IN PATIENTS WITH ADVANCED SOLID TUMORS}

${ }^{1} A$ ABBAS*, ${ }^{2}$ S Strauss, ${ }^{3} \mathrm{~F}$ Janku, ${ }^{4} \mathrm{R}$ Karim, ${ }^{5} \mathrm{~A}$ Olszanski, ${ }^{6} \mathrm{JJ}$ Luke, ${ }^{1} \mathrm{~K}$ Leach, ${ }^{1} \mathrm{R}$ lyer. ${ }^{1}$ Spring Bank Pharmaceuticals Inc, Hopkinton, MA, USA; ${ }^{2}$ Mary Crowley Medical Research Center, Dallas, TX, USA; ${ }^{3}$ MD Anderson Cancer Center, Houston, TX, USA; ${ }^{4}$ Next Oncology, San Antonio, TX, USA; ${ }^{5}$ Fox Chase Cancer Center, Philadelphia, PA, USA; ${ }^{6}$ UPMC Hillman Cancer Center, Pittsburgh, PA, USA

\subsection{6/jitc-2020-ITOC7.14}

Background Immunotherapy has emerged as a transformative approach for the treatment of cancer. However, a significant percentage of patients are nonresponsive to these immunotherapies or experience disease relapse which highlights the need for new therapies. Recent work has highlighted a major role for Stimulator of Interferon Genes (STING) agonists in immunotherapy. Conceptually, the activation of the STING pathway in immune cells in the tumor microenvironment (TME) and tumor cells could result in the induction of innate and adaptive immunity and subsequent activation of cytotoxic $\mathrm{T}$ cells and NK cells for durable anti-tumor responses. SB 11285 is a novel agonist of STING pathway leading to the activation of tumor-resident APCs and priming of tumor antigen specific $\mathrm{CD} 8+\mathrm{T}$ cells. In our preclinical studies using multiple tumor-derived cell lines, SB 11285 has been observed to cause the induction of cytokines, such as INF-b, INF-a, TNFa and others consistent with engagement of the STING target, as well as tumor cell death by STING-mediated apoptosis. SB 11285 reduced tumor volumes in multiple rodent tumor models when administered intravenously, intraperitoneally and intratumorally. Systemic administration could additionally facilitate trafficking of newly activated CD8+T cells from periphery into the tumor site. In addition, preclinical models indicate that survival and local tumor shrinkage were significantly enhanced when SB11285 was administered with anti-CTLA-4 or anti-PD-1 antibody, suggesting that SB 11285 can be administered with anti-PD-1 and anti-CTLA-4 antibody for synergistic activity. A multiple ascending dose, phase $1 \mathrm{a} / 1 \mathrm{~b}$ trial of SB11285 in multiple tumor types has been initiated and the objectives of this trial include determining a safe and efficacious dose of intravenous SB 11285 and a preliminary assessment of antitumor activity/efficacy as either monotherapy or in combination with nivolumab. 
Materials and Methods This open-label, multicenter phase 1a/ 1b clinical trial (NCT04096638) aims to enroll approximately 110 patients in the dose escalation (Part 1) and expansion cohorts (Part 2). Part 1 of the trial is a dose escalation study with IV SB11285 monotherapy followed by combination with the checkpoint inhibitor nivolumab. Part 1 Dose Escalation of the study will evaluate ascending doses of intravenously administered SB 11285 with respect to dose-limiting toxicities (DLTs), maximum tolerated dose (MTD), recommended phase 2 dose (RP2D) and the pharmacokinetic (PK)/pharmacodynamic profile as monotherapy and in combination with nivolumab. SB 11285, with a starting dose of $0.3 \mu \mathrm{g} / \mathrm{kg}$, will be administered as monotherapy weekly on Days 1, 8, 15, and 22 of repeated 28-day cycles in escalating doses and in combination with nivolumab administered on Q4W schedule. Part 2 Expansion Cohorts of the study will explore initial signs of efficacy in prespecified tumor types (such as Melanoma, HNSCC) using the recommended phase 2 dose (RP2D) of SB 11285 in combination with nivolumab. In addition, the biological effects of SB 11285 will be evaluated by changes in immune cell types and activation state, serum cytokines, and gene expression patterns indicative of activation of the immune compartment. The trial is being conducted at multiple sites in the U.S.

Disclosure Information A. Abbas: A. Employment (full or part-time); Modest; Spring Bank Pharmaceutical Inc. J. Strauss: E. Ownership Interest (stock, stock options, patent or other intellectual property); Modest; Abbvie, Abbott Laboratories, Bristol-Myers Squibb, Intuitive Surgical, Johnson \& Johnson, Merck. F. Consultant/Advisory Board; Modest; Tempus. Other; Modest; Dialectic Therapeutics. F. Janku: None. R. Karim: None. A. Olszanski: F. Consultant/Advisory Board; Modest; Bristol Myers Squibb. J.J. Luke: B. Research Grant (principal investigator, collaborator or consultant and pending grants as well as grants already received); Modest; All to institution for clinical trials unless noted) Abbvie, Bristol Myers Squibb, Medimmune, Necktar, Novartis, Merck, Leap, Incyte, Immunocore, Compugen, Corvus, Evil, Five Prime, Genentech, Immatic. F. Consultant/Advisory Board; Modest; Consultant: Akrevia, Algios, Array, Astellas,AstraZeneca, Bayer, Bristol Myers Squibb/Advisory Board:7 Hills, Actym, Alphamab Oncology, Mavu (now part of Abbvie), Pyxis, Spring Bank Pharma, Tempest. Other; Modest; Travel: Akrevia, Bayer, Bristol Myers Squibb, Reflexion, EMD Serono, Incyte, Janssen, Merck, Mersana, Novartis. K. Leach: A. Employment (full or part-time); Modest; Spring Bank Pharmaceuticals Inc. R. Iyer: A. Employment (full or part-time); Modest; Spring Bank Pharmaceuticals Inc.

\section{P01.02 HLA CLASS-I AND CLASS-II RESTRICTED NEOANTIGEN LOADS PREDICT OVERALL SURVIVAL IN BREAST CANCER}

${ }^{1} \mathrm{YW}$ Asmann*, ${ }^{1} \mathrm{Y}$ Ren, ${ }^{1} \mathrm{DP}$ Wickland, ${ }^{2} \mathrm{~V}$ Sarangi, ${ }^{2} \mathrm{~S}$ Tian, ${ }^{2} \mathrm{JM}$ Carter, ${ }^{2} \mathrm{AS}$ Mansfield, ${ }^{2}$ MS Block, ${ }^{1}$ ME Sherman, ${ }^{1} \mathrm{KL}$ Knutson, ${ }^{2} \mathrm{Y}$ Lin. ${ }^{1}$ Mayo Clinic, Jacksonville, $F L$, USA; ${ }^{2}$ Mayo Clinic, Rochester, MN, USA

\subsection{6/jitc-2020-ITOC7.15}

Background Tumors acquire numerous mutations during development and progression. These mutations give rise to neoantigens that can be recognized by $\mathrm{T}$ cells and generate antibodies. Tumor mutational burden (TMB) is correlated with, and has often been used as a surrogate of, neoantigen load, although that relationship is different depending on cancer types. Recent studies reported correlations between higher TMB and better overall survival after immune checkpoint blockade therapies in bladder, colorectal, head and neck, and lung cancers but not in breast cancer. On the other hand, the relationship between neoantigen load and survival has been controversial in literature. Higher neoantigen load has been linked to better overall survival in ovarian cancer and melanoma, but worse survival in multiple myeloma. Recently, no clear associations were found between neoantigen load and survival in 33 cancer types although only class-I restricted neoantigens were included.

Materials and Methods We developed a bioinformatics workflow, REAL-neo, for identification, quality control (QC), and prioritization of both class-I and class-II human leukocyte antigen (HLA) bound neoantigens that arise from tumor somatic single nucleotide mutations (SNM), small insertions and deletions (INDEL), and gene fusions. The correlations between TMB and neoantigen load per sample were calculated using Pearson Correlation Coefficient. TMB and neoantigen load comparisons between various groups were performed using Student's t-test. The survival analyses were performed using the Cox proportional hazards models while correcting for covariates.

Results We applied REAL-neo to 835 primary breast tumors in the Cancer Genome Atlas (TCGA) and performed comprehensive profiling and characterization of the predicted neoantigens. SNMs contributed to only $6.25 \%$ of the total neoantigens (\# of class-I vs. class-II neoantigens = 1: 3.5 ); INDELs accounted for $57.17 \%$ of the total (class-I : class-II= $1: 2$ ), and gene fusions were responsible for $36.58 \%$ of the total (class-I : class-II $=1: 2 \cdot 2$ ). TMB were positively correlated with total and each sub-categories of neoantigen load (class I: SNM: $\mathrm{r}=0.59, \mathrm{p}<2.2 \mathrm{E}-16$; INDEL: $\mathrm{r}=0.28, \mathrm{p}$ $<2.2 \mathrm{E}-16$; gene fusion: $\mathrm{r}=0.26, \mathrm{p}=2.01 \mathrm{E}-11$; class II: SNM: $r=0.47, p<2.2 \mathrm{E}-16$; INDEL: $r=0.16, p=1.7 \mathrm{E}-$ 05 ; gene fusion: $\mathrm{r}=0.31, \mathrm{p}=4.37 \mathrm{E}-13)$. The vast majority $(99.75 \%)$ of the predicted neoantigens occurred in $\leq 1 \%$ of the cases and $83.76 \%$ were patient-specific found in one patient only. Tumors with somatic and germline functional mutations in BRCA1 or BRCA2 genes had higher TMB ( $\mathrm{p}=$ 2.76E-06) and overall neoantigen load ( $p=0.009)$. Lower HLA class-I and class-II restricted neoantigen loads from SNM and INDEL were found to predict worse overall survival independent of TMB, breast cancer subtypes, tumor infiltrating lymphocyte (TIL) levels, tumor stage, and age at diagnosis (class-I: HR $=1.81, \mathrm{p}=0.04 ;$ class-II: HR $=1.89, \mathrm{p}=$ 0.042).

Conclusions Our study highlighted the importance of accurate and comprehensive neoantigen profiling and QC, and is the first to report the predictive value of neoantigen load for overall survival in breast cancer. This work was support by the State of Florida Cancer Center Grant, the bioinformatics program of Mayo Clinic Center for Individualized Medicine, and the Mayo Clinic inter-SPORE development grant.

Disclosure Information Y.W. Asmann: None. Y. Ren: None. D. P. Wickland: None. V. Sarangi: None. S. Tian: None. J.M. Carter: None. A.S. Mansfield: None. M.S. Block: None. M.E. Sherman: None. K.L. Knutson: None. Y. Lin: None. 